\title{
Agenda prospectiva de investigación de la cadena productiva de la panela y su agroindustria
}

\author{
Prospective research guidelines for the production chain \\ of sugarcane - (focus on panela, not centrifuged sugar)
}

\begin{abstract}
Diego Hernando Flórez Martínez
Ingeniero Químico, candidato a Magíster en Ingeniería Industrial. Docente Investigador de la Fundación Universitaria San Martín. Bogotá, Colombia.

Contacto:diego.florez@ingenieria.sanmartin.edu.co

Fecha de recepción: 28 de marzo de 2012

Clasificación del artículo: Investigación

Fecha de aceptación: 12 de febrero de 2013

Financiamiento: Ministerio de Agricultura y Desarrollo Rural
\end{abstract}

Palabras clave: agroindustria, benchmarking, cadena productiva, panela, prospectiva, tecnología.

Key words: agro-industry, benchmarking, productive chain, panela, prospective, technology.

\section{RESUMEN}

Con el objetivo de fortalecer el Sistema Nacional de Ciencia y Tecnología Agropecuaria, el Ministerio de Agricultura y Desarrollo Rural (MADR), a través del proyecto "Transición de la Agricultura”, inició en el año 2006 una serie de estudios con miras a la definición de las agendas prospectivas de investigación de 20 cadenas productivas. Esta iniciativa contempló, para el segundo periodo del 2008, la ejecución de la segunda fase de estudios, que incluyó a la cadena productiva de la panela y su agroindustria, en razón de su importancia económica y social, desarrollándose como un subsector dedicado a la generación de productos provenientes de la caña de azúcar, posicionándose, después del café, como el segundo renglón generador de empleo y, en quinto lugar, de los cultivos del país en términos de área cultivada. En este artículo se presentan los resultados del estudio cuyo objetivo fue la definición de la agenda prospectiva de investigación de la cadena, para lo cual se implementó una metodología enfocada a la articulación de herramientas de gestión tecnológica, como el análisis de desempeño, el benchmarking, la vigilancia tecnológica y comercial y la prospectiva a través de un sistema de inteligencia tecnológico, lográndose identificar cuatro áreas estratégicas para la investigación y desarrollo tecnológico de la cadena, que agrupan 11 demandas tecnológicas las cuales se proponen abordar a través de 41 bases para proyectos prioritarios enfocados al mejoramiento de la posición competitiva de la cadena productiva de la panela y su agroindustria. 


\section{investigación}

\section{ABSTRACT}

In order to strengthen the National System of Agricultural Science and Technology, the Ministry of Agriculture and Rural DevelopmentMARD (within the "Transition of Agriculture" project) launched a series of studies in 2006 aimed at defining Prospective Investigation-guidelines documentation for 20 productive chains. This initiative included the implementation of the second phase of the study by the second half of 2008, which involves the productive chain of sugar cane agro-industry focused on Panela due to its economic and social importance. In this context the Panela industry represents a sub-sector dedicated to the generation of products from sugar cane, positioning such products, right after coffee, as the second line of rural employment generation and occupies the fifth position in the country crops classification in terms of acreage (fields). This article presents the results of a study whose main purpose was to define prospective research guidelines for the productive chain by means of a conceptual and methodological perspective that involved the use of technological management tools such as performance analysis, benchmarking, technological monitoring, commercial market trends and foresight. This was achieved through a successful technology intelligence system, where four strategic areas for research and technological development were identified for the chain by grouping 11 technological needs that are to be addressed from 41 baselines linked to priority projects. These projects focus on improving the competitive position of the productive chain associated to sugar cane (particularly Panela) agro-industry.

\section{INTRODUCCIÓN}

Actualmente, el mundo se encuentra marcado por el cambio constante en todas las dimensiones de desarrollo y sostenibilidad, aunque estos cambios impactan de manera particular el entorno empresarial y de negocios, en razón de las dinámicas aceleradas de los mercados, la tecnología, las formas de gestión, las bases de la competitividad, entre otros factores que condicionan el desempeño de los sistemas productivos y establecen nuevos requerimientos en la generación de valor al interior de las organizaciones, así como directrices que orienten este cambio. Esta situación se presenta en todos los sectores de la economía, con características y particularidades para cada sector, y vectores de desarrollo más pronunciados y evidentes en actividades económicas transversales, como en el caso de las tecnologías de información y comunicaciones y los sectores basados en conocimiento.
En economías emergentes, como la colombiana, se hace necesario explorar las dimensiones e implicaciones del cambio actual en uno de los sectores de mayor importancia social y económica: el sector agrícola. En Colombia, este sector es de gran relevancia económica debido a que es una de las cinco primeras actividades que más contribuyen al Producto Interno Bruto (PIB) y hace un significativo aporte a otros sectores, como la industria manufacturera, a través del suministro de materias primas y servicios, constituyéndose en uno de los pilares sobre los cuales se desarrolla económicamente el país y en el cual se involucra buena parte de su población rural e industrial.

En lo que respecta a la Cadena Productiva de la Panela y su Agroindustria, ésta es una muestra de la integración donde el encadenamiento de actividades de producción agrícola, transformación, comercialización intermedia y final, y consumo, son desarrollados por distintos sistemas producti- 
vos que se ubican en sectores diversos de la economía, como el agrícola, el manufacturero y el de servicios.

La caña de azúcar de la familia (Gramineae), género especie (Saccharumofficinarum), presenta variedades cuyas condiciones y características son óptimas para su aprovechamiento en la producción de panela, estas variedades se denominan, de manera genérica, caña panelera en Colombia. La panela, denominada por la Organización de las Naciones Unidas para la Agricultura y la Alimentación (FAO), como azúcar no centrifugado, es un alimento típico de Brasil, Perú, México, Centro América, Panamá, Colombia, Venezuela, Ecuador y Bolivia, cuyo único ingrediente es el jugo de la caña de azúcar, también es producida en algunos países asiáticos, como la India y Pakistán. Las características y composición como alimento, edulcorante, complemento alimentico y su origen natural, ofrecen posibilidades en la apertura de nuevos mercados por medio de un estudio integral de la cadena productiva (MADR, 2006).

Para producir la panela, el jugo de caña de azúcar es cocido a altas temperaturas hasta formar una melaza bastante densa, luego, se pasa a unos moldes en forma de cubo donde se deja secar hasta que se solidifica o cuaja. La FAO registra la panela en sus cuentas como "azúcar no centrifugado" [8]. El mercado actual en que se encuentra desarrollada la actividad productiva de la cadena de la panela y su agroindustria, se enmarca en el concepto mismo que maneja el cliente del producto a nivel nacional e internacional; donde se percibe como un edulcorante de origen natural o un alimento, complemento básico del contenido nutricional requerido en la dieta de la población nacional [9]. La producción de panela es una de las agroindustrias rurales de mayor tradición en América Latina y el Caribe. En contraste a la industria azucarera, la producción de panela se realiza en pequeñas explotaciones campesinas, mediante procesos artesanales en los que prevalece una alta intensidad de trabajo familiar y aún muy bajas tasas de introducción de tecnologías mecanizadas o de alta intensidad de capital [13].

El estudio para la definición de la agenda de investigación que se inició a mediados del año 2008, con el apoyo del Ministerio de Agricultura y Desarrollo rural y la asesoría metodológica de consultores brasileños reconocidos a nivel internacional, se realizó con el objetivo de aumentar la competitividad de los sectores agropecuario y agroforestal colombiano, a partir del mejoramiento de los procesos de investigación y desarrollo tecnológico para las cadenas asociadas al agro y, de este modo, poder contribuir al fortalecimiento del Sistema Nacional de Ciencia y Tecnología Agropecuaria.

\section{METODOLOGÍA}

Durante las últimas dos décadas del siglo pasado, la gestión tecnológica se formuló en sistemas y procesos de aprendizaje, fortaleciéndose los mecanismos de desarrollo tecnológico, como la innovación y la transferencia de tecnología. Así mismo, la consolidación de la gestión de tecnología evidenció la prioridad otorgada a la generación y aplicación de conocimiento en los procesos productivos, lo que se reflejó en nuevos productos y bienes de capital implementados en la producción. Según [2], a pesar de la inserción de la gestión tecnológica en países con economías emergentes, sus dinámicas se retrasaron, en algunos casos hasta en diez años, respecto de los avances generados en las economías líderes en el mundo.

En la actualidad, el sector agroindustrial debe tomar sus decisiones en forma eficaz y eficiente para responder al ritmo con el cual las tecnologías entran al mercado y jugar un papel fundamental en la obtención de productos de calidad que sean altamente competitivos. En este sentido, la gestión de tecnología ha evolucionado, y si bien reto- 


\section{investigación}

ma las bases y el valor de las etapas anteriores de su desarrollo, en la actualidad se proyecta desde la gestión de la información y su transformación en conocimiento, considerado como motor impulsor de la productividad y el desarrollo económico del siglo XXI.

\subsection{Herramientas de gestión tecnológica}

Las ventajas ofrecidas por las herramientas de gestión tecnológica se potencializan cuando se tienen claros, no solo los objetivos de utilizarlas sino, además, se visualizan los posibles resultados que se quieren lograr. En este sentido, se describirán a continuación algunas herramientas de gestión tecnológica, incluyendo la prospectiva tecnológica.

Diagnóstico tecnológico, como punto de partida para la identificación de oportunidades y limitaciones de la agroindustria. En una cadena agroindustrial es imprescindible entender la problemática que afronta esta e identificar las necesidades generales. Todo lo que la cadena hace implica tecnología de algún tipo, la cual tiene un impacto importante sobre factores de calidad y competitividad [12]. Los análisis de información secundaria, a partir de estudios de diagnóstico anteriores, permiten establecer la evolución y el estado actual de las variables que determinan el desempeño de los sistemas productivos, establecer tendencias y factores críticos que obstaculizan o potencializan la competitividad de la cadena productiva. En este sentido, el análisis de documentos y tendencias obtenidos de la recopilación de información secundaria, contribuye enormemente a la caracterización de la cadena productiva. Así mismo, las deficiencias encontradas en este análisis, permiten y justifican la planeación y realización de trabajos de campo que tienen como fin complementar y validar información valiosa para el análisis de la cadena, lo que es considerado como recopilación de información primaria.
Benchmarking, como herramienta de comparación del desempeño de la agroindustria. La expresión inglesa benchmarking significa "punto de referencia", es decir: algo que sirve como una norma (referencia) contra la cual otras cosas son comparadas y medidas. Es un conjunto de procedimientos mediante los cuales, sectores como la agroindustria, comparan su desempeño, contra su propio desempeño pasado, o contra el de otros sectores comparables [10], revisando prácticas, procesos, productos, servicios, políticas e indicadores de las organizaciones que, por tener las mejores prácticas, son consideradas líderes, para poder identificar, adaptar e implementar estrategias que permitan el mejoramiento del desempeño empresarial. Sin embargo, como se explica en [1], solo la reproducción de avances de los competidores no basta para garantizar posición competitiva favorable, es necesario el desarrollo de capacidad innovadora.

Vigilancia tecnológica y comercial en el conocimiento de tendencias en tecnologías y mercados. La importancia de la vigilancia tecnológica en el contexto de las economías emergentes es entendida, cada vez más, como el esfuerzo realizado por un sector para la búsqueda, análisis y difusión de información científica y tecnológica, permitiendo la identificación de tendencias emergentes y decadentes en el desarrollo tecnológico, lo cual prepara a los sistemas productivos para anticiparse a los cambios [7]. De esta forma, los sectores pueden establecer dinámicas en el desarrollo tecnológico y científico de las áreas de interés, incorporando, a sus actividades de investigación, desarrollo e innovación, elementos que permitan seguir tendencias que mejoren su posicionamiento en el mercado actual. De este modo, la identificación de líderes, tendencias y redes de investigación, aportará bases para definir estrategias de acortamiento en las brechas tecnológicas de la cadena productiva, diferenciando entre las tecnologías de base, clave, las de paso y las emergentes, entre otras. Las tecnologías de paso y las 
emergentes son en extremo fundamentales, debido a que pueden llegar a ser más significativas en un marco estratégico. Por otro lado, la vigilancia comercial brinda elementos importantes para la generación de ventajas competitivas, así como para el análisis de la competitividad de una actividad productiva. El análisis del mercado permite, a los sistemas productivos, conocer el estado actual de la actividad comercial en el propio sector económico donde se desenvuelve y también, de industrias relacionadas de manera directa e indirecta. Un análisis como este permite establecer tendencias de mercado, principales competidos, líderes del mercado y perfiles empresariales, para brindarle a la cadena algunas posibilidades que potencialicen la comercialización de los productos existentes o las bases para incursionar con productos innovadores.

Prospectiva tecnológica, como herramienta estratégica en la gestión de la innovación. Esta herramienta ha sido ampliamente definida, estudios realizados por varios autores como [7], [6] y [11], pueden afirmar que está constituida por un conjunto de análisis y estudios encaminados a explorar o "predecir", con cierto nivel de confianza, posibles estados futuros de la tecnología y su influencia en un determinado sector, de tal manera que ayude a comprender cuáles son las variables que pueden modelar su futuro a largo plazo. La prospectiva tecnológica no propone una única solución o un único escenario futuro, han de estar presentes varias posibles soluciones, hasta, quizás, quedar reducida a una única, según vaya avanzando el tiempo. Algunos de estos escenarios posibles pueden ser una opción no deseable y, por ende, se presenta la oportunidad de poder tomar todas las medidas posibles para que no llegue a ser una realidad en el futuro [11]. En esta metodología de gestión tecnológica se reconocen y se manejan herramientas que permiten la exploración de las fuentes de información, primaria o secundaria, para identificar la evolución de los factores y variables que determinan el desarrollo del sistema agroindustrial prospectado. El análisis prospectivo de cadenas productivas requiere el establecimiento de un conjunto de herramientas que se ajusten a la complejidad y la especificidad del sistema bajo análisis, el conocimiento de los expertos del sector en las técnicas prospectivas, así como de los alcances y tiempos de ejecución del estudio.

\subsection{Agenda de investigación}

La construcción de la agenda de investigación implicó un análisis integral de la información obtenida a partir de cada una de las herramientas de gestión implementadas en el proceso. Cada una de estas herramientas aportó elementos valiosos para la definición de la agenda de investigación, los cuales se sintetizan en la tabla 1 .

La articulación de estos aportes se consolidan en las demandas identificadas y en el escenario definido para la cadena; a través de la agenda se da respuesta a las demandas considerando dos componentes esenciales: 1) los lineamientos tecnológicos que hacen referencia a un conjunto de proyectos cuyo propósito es el fortalecimiento de las demandas tecnológicas, y 2) los lineamientos organizacionales que abarcan las estrategias o acciones encaminadas a suplir las demandas no tecnológicas. Para la identificación de estos lineamientos tecnológicos se trabajó un instrumento, en el cual los actores de la cadena definen, para cada una de las demandas tecnológicas, los proyectos, incluyendo el plazo (corto, mediano o largo) en que deberían ser implementados para que su impacto sea tangible en el horizonte definido para el análisis prospectivo, y definían además posibles responsables y mecanismos de seguimiento. Las demandas tecnológicas están organizadas en áreas estratégicas de trabajo investigativo y de desarrollo tecnológico donde se centralizan la generación de valor para el sector, éstas han sido definidas por el Ministerio de 
Tabla 1. Aporte de cada herramienta del sistema a la definición de la agenda de investigación de la panela y su agroindustria

\begin{tabular}{|c|c|}
\hline HERRAMIENTA & APORTE A LA DEFINICIÓN DE LA AGENDA \\
\hline Análisis de desempeño & $\begin{array}{l}\text { - Caracterización del agronegocio de los edulcorantes afines a la panela a nivel mundial. } \\
\text { - Caracterización del agronegocio de la panela y de su cadena productiva a nivel nacional. } \\
\text { - Conocimiento del desempeño de cada uno de los eslabones de la cadena productiva de la panela. } \\
\text { - Establecimiento y comprensión de la situación actual del sector. } \\
\text { - Determinación de oportunidades y limitaciones para cada uno de los eslabones de la cadena. }\end{array}$ \\
\hline Benchmarking & $\begin{array}{l}\text { - Identificación de los entornos competidoras de la panela (rapadura, jaggery, gur, Chancaca, azú- } \\
\text { car muscovado, piloncillo) y los países productores (India, Brasil, Filipinas, isla Mauricio, México } \\
\text { y Ecuador). } \\
\text { - Comparación con los otros entornos competidores (Jaggery en India, rapadura en Brasil, chanca- } \\
\text { ca en Ecuador y Perú , piloncillo en México, azúcar moscabado en isla Mauricio y Filipinas. } \\
\text { - Identificación de la mejor práctica en ambiente organizacional e institucional. }\end{array}$ \\
\hline Vigilancia tecnológica & $\begin{array}{l}\text { - Orientación sobre tecnologías (maquinaria y procesos) en diversidad genética, manejo de cultivo, } \\
\text { extracción del jugo de caña, producción de nuevos productos y derivados de la panela. } \\
\text { - Investigación básica y aplicada en el sector a nivel mundial Desarrollo tecnológico en el sector } \\
\text { mundialmente. } \\
\text { - Capacidades nacionales en investigación y desarrollo tecnológico sobre panela. } \\
\text { - Establecimiento de las brechas en proceso y productos. }\end{array}$ \\
\hline Vigilancia comercial & $\begin{array}{l}\text { - Orientación sobre los productos derivados de la caña que se comercializan a nivel mundial; perfi- } \\
\text { les de las empresas que participan en la comercialización, presentaciones y precios manejados, } \\
\text { importaciones y exportaciones. } \\
\text { - Orientación sobre tendencias filtración de jugo de caña, nuevos productos elaborados del jugo y } \\
\text { panela como insumo de otras industrias. } \\
\text { - Principales tendencias de los mercados. } \\
\text { - Caracterización de los mercados reales y potenciales para los productos de panela priorizados a } \\
\text { nivel nacional e internacional. } \\
\text { - Retos comerciales. }\end{array}$ \\
\hline Prospectiva tecnológica & $\begin{array}{l}\text { - Identificación de factores críticos de competitividad para la cadena de la panela y su agroindustria. } \\
\text { - Determinación de la influencia de los factores críticos en el estado actual. } \\
\text { - Importancia y gobernabilidad de los factores críticos en un futuro. } \\
\text { - Establecimiento del escenario apuesta hacia cual se deben orientar los esfuerzos y recursos de } \\
\text { la cadena para hacerla más competitiva-sostenible. } \\
\text { - Establecimiento de la influencia de los actores en los factores críticos. }\end{array}$ \\
\hline
\end{tabular}

Fuente: elaboración propia.

Agricultura y Desarrollo Rural en el marco de los Fondos Concúrsales. Así mismo, el instrumento incluyó la definición de estrategias que, posteriormente, se constituyeran en los lineamientos organizacionales e institucionales. De este modo, la agenda surge de un proceso de socialización y articulación con expertos de la cadena, quienes cuentan con una amplia trayectoria en el sector, en el cual plantean aquellos proyectos y estrategias necesarios para abordar las demandas.

Metodología: en Colombia, los actores de la cadena productiva de la panela y su agroindustria se enfrentan a numerosos retos, entre los que sobre- salen: la introducción de nuevas tecnologías que permitan el mejoramiento de los tradicionales procesos productivos; el desarrollo de productos innovadores y diferenciados que permitan una mayor competitividad; la consolidación de un tejido social de la cadena, que procure la focalización de esfuerzos adelantados en las trece regiones paneleras y evite la atomización de oferta y demanda, con condiciones inequitativas entre los diferentes eslabones de la cadena. En este sentido, la formulación de la agenda prospectiva de investigación y desarrollo surge en un momento apropiado para buscar soluciones a problemáticas tecnológicas y aquellas que tienen una fuerte in- 
fluencia en la sostenibilidad de la cadena, como el aumento en las áreas cultivadas, en parte como consecuencia de las expectativas generadas con el montaje de plantas de bioetanol, la baja en los precios de la panela en bloque, como resultado de la sobreoferta con características de calidad de marcadas diferencias, entre otros aspectos.

Para la definición de la Agenda de Investigación de la cadena productiva de la panela y su agroindustria, se tomaron como referencia los fundamentos planteados por los consultores brasileros en lo relacionado con análisis de desempeño de cadenas productivas y análisis prospectivo, y la experiencia del equipo ejecutor del estudio el Grupo de Investigación y Desarrollo Bio Gestión de la Universidad Nacional de Colombia, en la implementación y articulación de herramientas de gestión tecnológica para el direccionamiento estratégico. El resultado fue una metodología que incluyó cuatro etapas fundamentales: en primer lugar, se estableció el contexto del estudio, identificándose el problema por abordar, los límites del estudio y los objetivos de este y un marco conceptual y metodológico; en segundo lugar, se realizó un análisis de la cadena productiva de la panela y su agroindustria, aplicando un enfoque sistémico se modeló, representó y describió la cadena en términos de los eslabones y segmentos, adicionalmente, se analizó el desempeño de la cadena mediante la caracterización del agronegocio de la panela y sus homólogos a nivel nacional e internacional, utilizando herramientas de gestión tecnológica como benchmarking, vigilancia tecnológica y vigilancia comercial, para lograr obtener los insumos necesarios con el objetivo de definir comportamiento del sistema en el pasado y el presente, y los factores que causaron su desempeño; en tercer lugar, se realizó un análisis prospectivo que buscaba determinar el comportamiento futuro de los factores críticos definidos en el análisis de la cadenas, lográndose identificar cuatro estados futuros posibles para la cadena; y finalmente, se realizó la definición de la agenda prospectiva de investigación de la cadena, la cual se enfocó en un producto priorizado debido al amplio espectro de productos que pueden ser elaborados y sintetizados a partir de la caña de azúcar (S. officinarum) como materia prima de la actividad productiva de la cadena, con base en los resultados del análisis de la cadena y la prospectiva, realizando un proceso constante de validación y socialización con los actores de la cadena.

\section{RESULTADOS}

\subsection{Análisis de desempeño y prospectivo de la cadena productiva de la panela y su agroindustria}

Para la representación y análisis de la cadena productiva, se realizó una segmentación de los eslabones de la cadena, dicha segmentación se sustenta en la definición y observación de ciertas variables asociadas a los sistemas productivos, como tamaño, nivel de adopción tecnológica, ubicación geográfica, mercado al que atiende, entre otros; además, se contemplan los componentes o instituciones que se encuentran en la cadena productiva y están relacionados con un ambiente institucional (leyes, normas e instituciones encargadas de formularlas, entre otras) y con un ambiente organizacional (entes gubernamentales y no gubernamentales, reguladores, entre otros) que ejercen una influencia importante sobre su desempeño $\mathrm{y}$, que por lo tanto, según [5], deben ser parte de su modelación.

El modelo de cadena planteado en el presente estudio, toma como insumo la información de estudios previos de entidades como el Ministerio de Agricultura y Desarrollo Rural, el Ministerio del Medio Ambiente, el Instituto Interamericano de Cooperación para la Agricultura-IICA, Corpoica $\mathrm{y}$ de los centros provinciales de gestión agroempresarial (CPGA), así como de las corporaciones autónomas regionales (CAR) de las cuatro regiones contempladas para el estudio. El modelo de la 


\section{investigación}

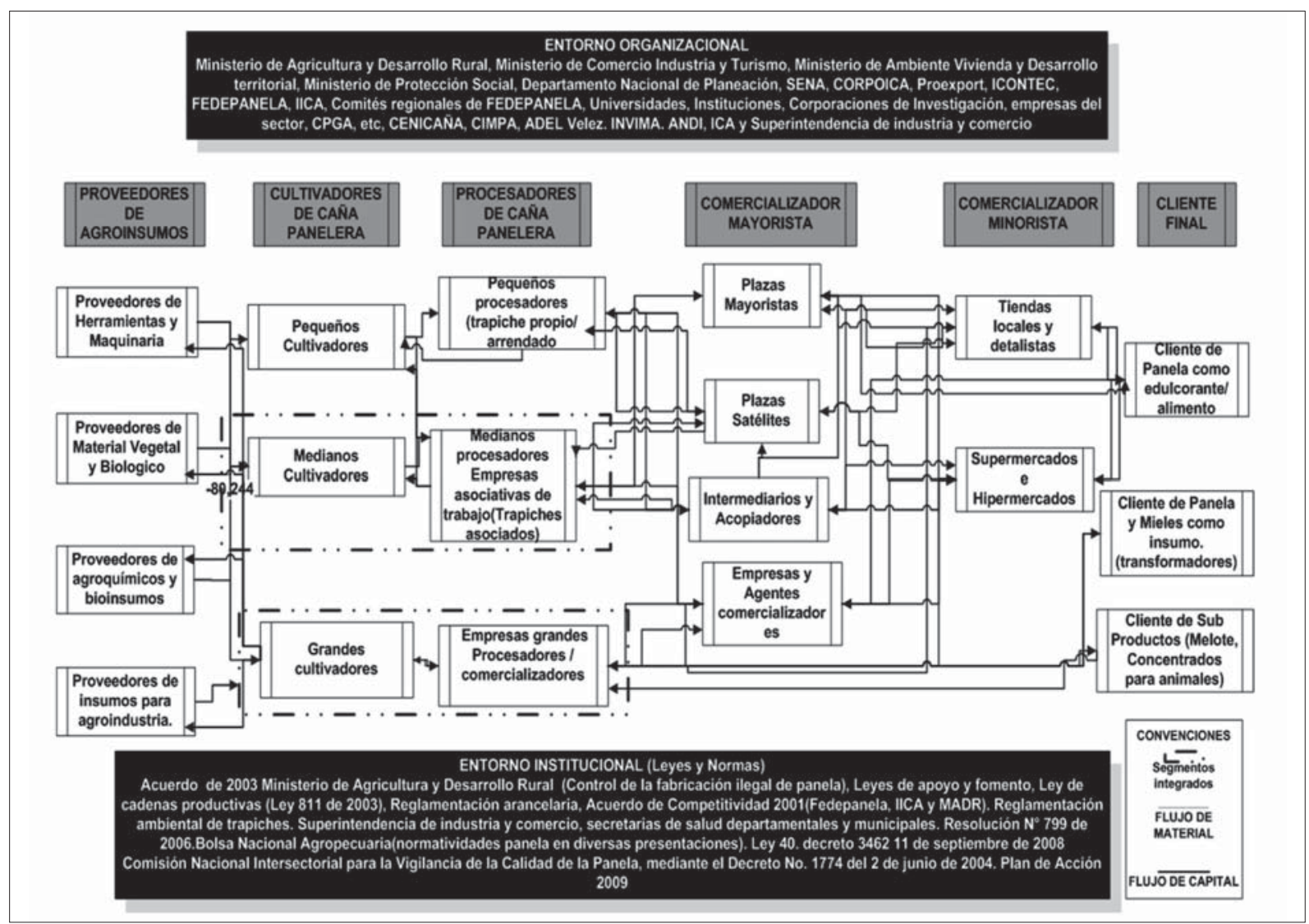

Figura 1. Modelo propuesto para la representación de la Cadena Productiva de la Panela y su Agroindustria Fuente: tomada de [4].

figura 1, el cual fue validado por los actores de la cadena, constituyó el punto de referencia para el proceso analítico de las diferentes características y necesidades de los eslabones y segmentos considerados, para establecer un mercado de tecnología, variables de adopción tecnológica entre otros elementos que encaminaron la formulación de la agenda de investigación.

En el modelo de la cadena productiva de panela y su agroindustria (figura 1), se identifican flujos de material y flujos de capital, esto debido a que, el flujo de material está directamente relacionado con el flujo de capital ya que el suministro de materia prima o de producto, implica necesariamente una retribución económica. El modelo costa de seis eslabones, los cuales hacen referencia a los actores principales de la cadena: clientes finales, comercializadores minoristas y proveedores de insumos, así como las relaciones entre ellos. Adicionalmente, el modelo considera el entorno de la cadena (ambiente institucional y organizacional). El modelo permite analizar el desempeño de la cadena productiva en términos de eficiencia y calidad, principalmente a partir de los costos que implican la actividad productiva, desde el cultivo de caña hasta llegar a la panela como producto final integrando; los costos por insumos, mano de obra, costo de la actividad y costos indirectos, lo cual permite establecer a nivel regional diferencias en la actividad, brechas, oportunidades y limitaciones. En la tabla 2 se presentan, por eslabón, las principales oportunidades y limitaciones identificadas, las cuales son el punto de partida 


\section{investigación}

Tabla 2. Oportunidades y limitaciones para la cadena productiva de la panela y su agroindustria

\begin{tabular}{|c|c|c|}
\hline Eslabón & Oportunidad & Limitación \\
\hline $\begin{array}{l}\text { Proveedores de } \\
\text { agroinsumos }\end{array}$ & $\begin{array}{l}\text { Existencia de investigaciones internaciona- } \\
\text { les en productos fertilizantes y biopesticidas } \\
\text { para las etapas de sostenimiento de cultivo. }\end{array}$ & $\begin{array}{l}\text { Bajo interés por fortalecer la oferta debido a una } \\
\text { baja demanda por parte de los productores. }\end{array}$ \\
\hline $\begin{array}{l}\text { Cultivadores de } \\
\text { caña panelera }\end{array}$ & $\begin{array}{l}\text { Alto impacto social debido a la generación } \\
\text { de empleo para el manejo y adecuación } \\
\text { del cultivo. } \\
\text { Desarrollo de hectáreas experimentales } \\
\text { para manejo de cultivo y definición de } \\
\text { necesidades agronómicas para el suelo. }\end{array}$ & $\begin{array}{l}\text { No se han definido las mejores variedades de caña } \\
\text { para la obtención de panela pulverizada. } \\
\text { Existencia de rendimientos disimiles en una misma } \\
\text { región debido a la heterogeneidad de variedades } \\
\text { cultivadas. }\end{array}$ \\
\hline $\begin{array}{l}\text { Procesadores de } \\
\text { caña panelera }\end{array}$ & $\begin{array}{l}\text { Iniciativas para la conformación de sistemas } \\
\text { asociativos productivos ha permitido me- } \\
\text { jorar los rendimientos de producción de } \\
\text { la panela tradicional así como la adapta- } \\
\text { ción de tecnologías para trascender a la } \\
\text { producción de panela pulverizada. } \\
\text { Capacidad de manejar canales de comer- } \\
\text { cialización directos con minoristas y } \\
\text { cliente final. }\end{array}$ & $\begin{array}{l}\text { La prórroga para la normatividad existente afecta } \\
\text { las dinámicas del mercado por cuanto desfavo- } \\
\text { rece a las panelas de buena calidad con respe- } \\
\text { to a panelas con baja calidad. } \\
\text { Falta de rigurosidad en el cumplimiento de las nor- } \\
\text { mas del sector afecta su comercialización y ca- } \\
\text { lidad. }\end{array}$ \\
\hline $\begin{array}{l}\text { Comercializador } \\
\text { mayorista }\end{array}$ & $\begin{array}{l}\text { Generación de asociaciones de producto- } \\
\text { res para el establecimiento de un canal } \\
\text { de comercialización directo entre los co- } \\
\text { mercializadores minoristas y el produc- } \\
\text { tor, eliminando los factores de interme- } \\
\text { diación. } \\
\text { Nexos comerciales directos con supermer- } \\
\text { cados de cadena para la panela como } \\
\text { alimento y con la industria de alimentos. }\end{array}$ & $\begin{array}{l}\text { El canal tradicional de comercialización sigue te- } \\
\text { niendo los mayores volúmenes de comercializa- } \\
\text { ción de panela en términos de oferta y demanda. } \\
\text { Falta integración directa entre los productores de } \\
\text { panela y los comercializadores mayoristas. }\end{array}$ \\
\hline $\begin{array}{l}\text { Comercializador } \\
\text { minorista }\end{array}$ & $\begin{array}{l}\text { Condiciones de comercialización estables } \\
\text { en volúmenes de compra y precios ofre- } \\
\text { cidos para agentes comercializadores y } \\
\text { procesadores de panela. } \\
\text { Mejores márgenes de utilidad en productos } \\
\text { de valor agregado }\end{array}$ & $\begin{array}{l}\text { Número de proveedores de panela limitado. } \\
\text { Heterogeneidad en productos de panela disponi- } \\
\text { bles en cuanto a calidad, color, peso neto y em- } \\
\text { paque. }\end{array}$ \\
\hline Cliente final & $\begin{array}{l}\text { Precios diferenciados para productos de } \\
\text { alta calidad y valor agregado. } \\
\text { Precios diferenciados para productos con } \\
\text { sellos de calidad, sellos orgánicos, sellos } \\
\text { verdes y de comercio justo. }\end{array}$ & $\begin{array}{l}\text { No se cuenta con la caracterización de mercados } \\
\text { y requisitos de estos para la panela pulverizada } \\
\text { como producto de exportación. }\end{array}$ \\
\hline
\end{tabular}

Fuente: elaboración propia.

80 Tecnura | Vol. 17 | No. 36 | abril - junio de 2013 


\section{investigación}

para los procesos de búsqueda del estado del arte en investigación nacional e internacional para la cadena.

\subsection{Benchmarking}

Con el objetivo de evaluar y establecer puntos de referencia para Colombia, este análisis endógeno de la cadena se complementó con la exploración del agronegocio y la realización de un benchmarking organizacional e institucional, así como el análisis de la cadena competidora. El agronegocio y el benchmarking tomaron como referentes: el jaggery o gur, en India; la rapadura y azúcar muscovado, en Brasil; el piloncillo, en México; la chancaca, en Perú y Ecuador; y los azúcares orgánicos demerara y muscovado, en Filipinas e Isla Mauricio. Se evaluaron aspectos como: la importancia del sector en los países de referencia, las políticas estatales y de regulación, el ambiente organizacional y de integración, las principales problemáticas y las condiciones del sector productivo, junto con aspectos de la cadena competidora referente en el contexto latinoamericano, como es Brasil, y en el contexto mundial India. Los referentes estudiados permitieron definir aspectos claves a nivel productivo, normativo y organizacional.

India: su entorno productivo es líder en el mercado mundial de la panela, y presenta un claro enfoque a la mejora de la competitividad en sus políticas para exportación y el grado de institucionalidad que se maneja en el sector agrícola; de igual manera, es el líder en investigación básica y aplicada en temáticas referentes al proceso productivo y productos diferenciados.

Filipinas: el muscovado se ha identificado en el Plan de Desarrollo de las Exportaciones de Filipinas 2005-2010, como uno de los productos promisorios, debido a la creciente demanda de este producto en la Unión Europea y Japón. Existen

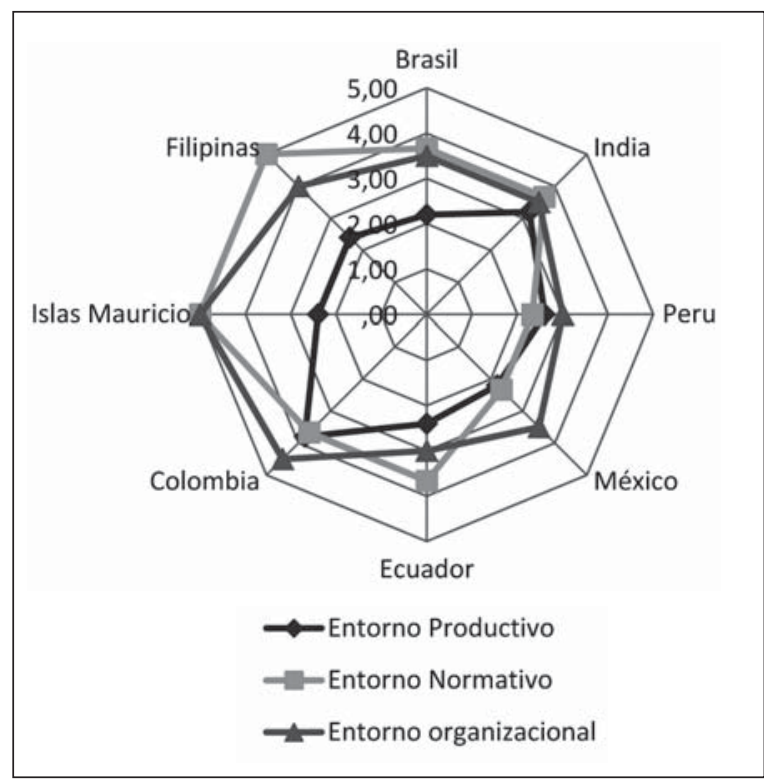

Figura 2. Resultado del proceso evaluativo de Benchmarking para la cadena productiva de la panela y su agroindustria frente a los entornos competidores

Fuente: elaboración propia.

múltiples instituciones que trabajan en pro de generar estándares para mejorar la calidad, y en la creación de programas de mejora del sistema productivo. Hay grandes asociaciones que, con el apoyo de entidades estatales, comercializan el producto en los mercados de la Unión Europea y Asia.

Brasil: la identificación de Brasil como referente competidor en la diversificación y fortalecimiento de la cadena productiva, a partir de políticas claras de integración y apoyo al sector para el mejoramiento de los nexos comerciales y el desarrollo tecnológico, lo convierten en el referente más próximo para Colombia; dada la diversificación que tiene la cadena azucarera en Brasil, así como la importancia directa que, en la economía del país, han generado el desarrollo de programas de fortalecimiento a la competitividad, fortalecimiento del desarrollo tecnológico y estratégico en todo el espectro de actividades. 
Cabe resaltar el desempeño de los entornos de Colombia, Brasil e India son de mejores resultados, siendo de estos últimos, junto con el caso de la Asociación Central Piurana de Cafetaleros (Cepicafe), los que el entorno colombiano debería adoptar las prácticas y políticas que sitúan a estos países como los referentes en el sector.

\subsection{Vigilancia tecnológica y comercial}

Con la implementación de herramientas como la vigilancia tecnológica y comercial se logró: 1) es- tablecer el estado del arte en investigación básica y aplicada, y en el desarrollo tecnológico de las principales compañías en el ámbito mundial; 2) determinar el alcance de la investigación en el país y su relación con las tendencias mundiales; y 3) evaluar las oportunidades de la cadena en términos comerciales, a partir de las tendencias que presenta el mercado mundial de los productos finales de la cadena. La investigación y el desarrollo tecnológico, en la cadena productiva, presentan un comportamiento creciente en términos de los resultados de estos procesos, documentados de manera formal mediante artículos científicos y patentes.

Tabla 3. Descripción de las dinámicas por área de investigación en artículos

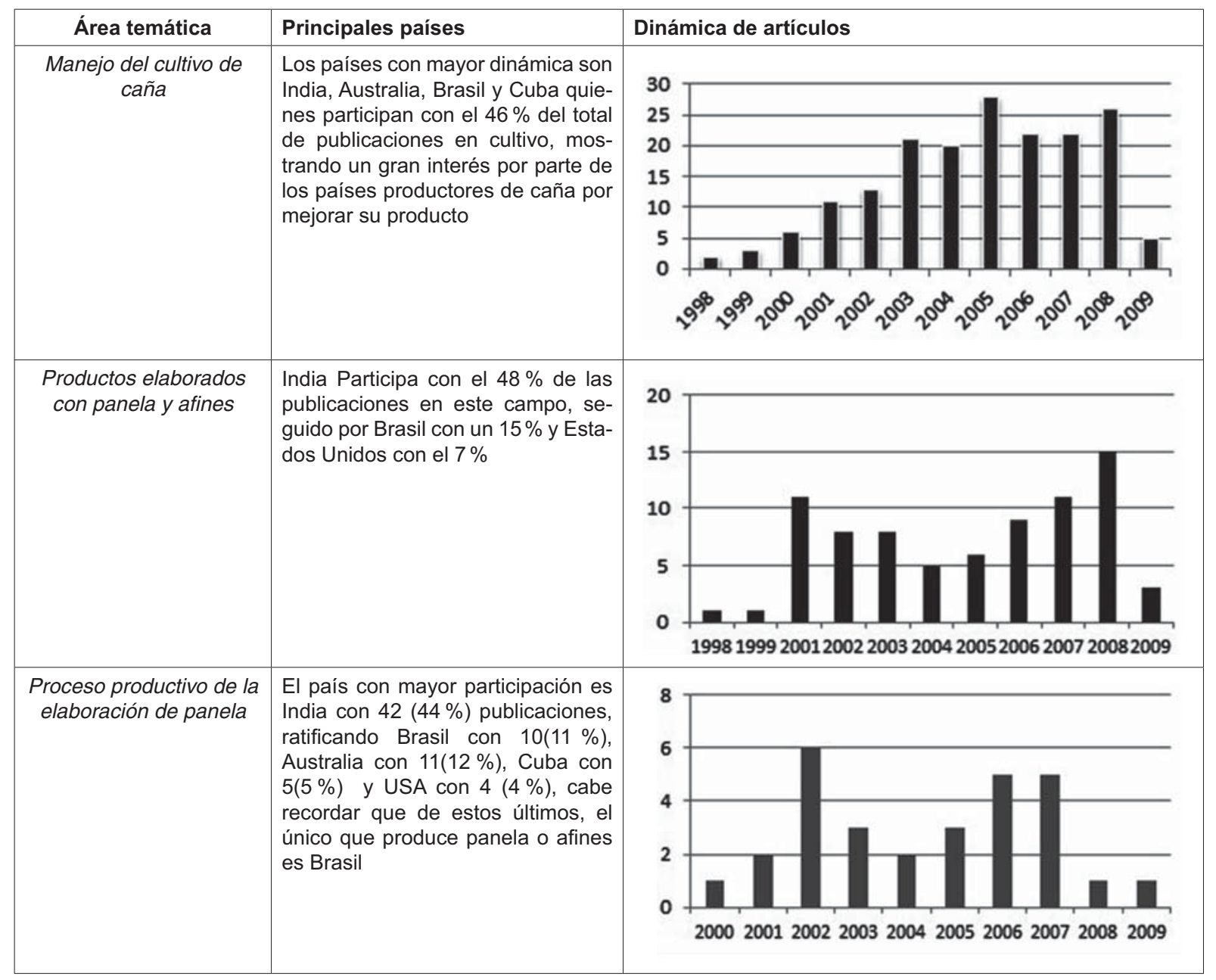

Fuente: tomada de [4]. 
Del análisis de la información encontrada en estas temáticas, se logró establecer que el dominio de la Investigación básica y aplicada en instituciones académicas, como universidades en el ámbito mundial, revela la importancia de las alianzas estratégicas entre instituciones de investigación y el sector productivo, una lección que aprender para Colombia, teniendo en cuenta que existe desarticulación entre la investigación realizada principalmente por instituciones académicas y los requerimientos de las empresas. En la tabla 3, se presenta la dinámica de artículos para las áreas temáticas identificadas, así como los principales países líderes en la investigación.

En la tabla 4, se realiza la descripción de las dinámicas para las tres temáticas establecidas en patentes.

Tabla 4. Descripción de las dinámicas por área de investigación en patentes

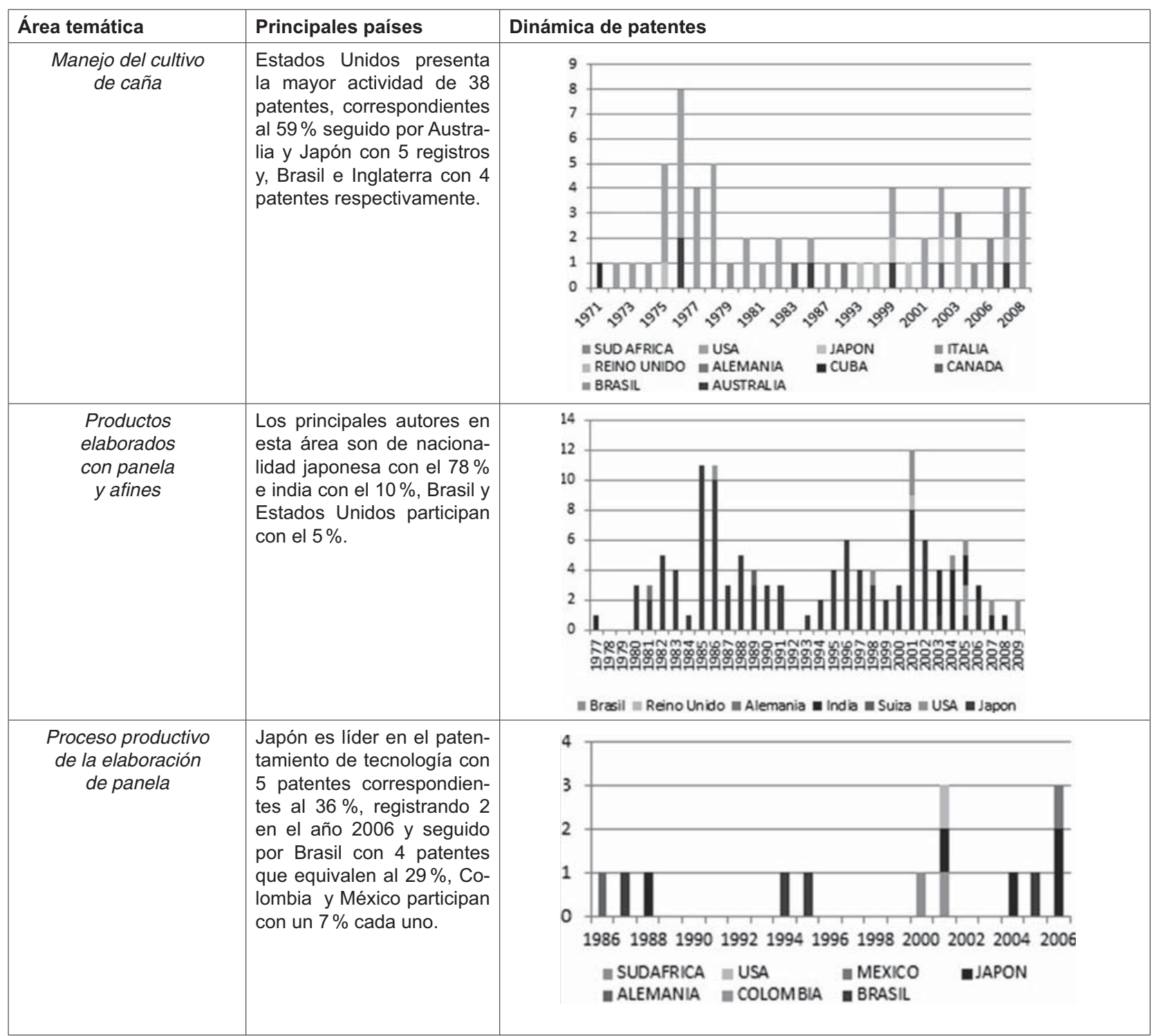

Fuente: tomada de [4]. 
De acuerdo con los resultados del estudio, cada país tiene un enfoque de investigación diferente, el cual se evidencia en sus publicaciones científicas y patentes realizadas; en el caso de India, este puede ser un referente a nivel productivo, en la formulación de productos diferenciados, así como en el mejoramiento de la etapas del procesos productivo enfocándose en una mayor calidad. Japón, es un referente en el patentamiento del uso de derivados de caña orgánicos en productos alimenticios y bebidas; Estados Unidos, es un ejemplo en el desarrollo de maquinaria para cultivo y mejoramiento genético de variedades, según condiciones climatológicas y agronómicas, al igual que Brasil.

En el ámbito comercial, teniendo en cuenta que se tiene a la panela pulverizada como el producto objeto de estudio para la agenda, es necesario especificar la dinámica comercial de la panela como producto global, identificando mercados potenciales para la cadena productiva (ver figura 3 ).

El diagrama de burbujas permite clasificar los mercados conocidos como importadores en po- tenciales, entendidos como aquellos que presentan un crecimiento favorable en la cantidad de producto comprado y reales mercados con un crecimiento estable de las importaciones. El eje vertical del gráfico, representa el crecimiento del mercado en porcentaje para la cantidad en kilogramos exportada, el eje horizontal el valor promedio para cada país del FOB unitario en Dólares, y el radio de la burbuja el porcentaje de mercado que no abarcan los productos de Colombia. Los mercados más promisorios para la panela son: Canadá, Reino Unido, España y Alemania. Actualmente, se estudian, por parte de Proexport, estrategias comerciales para los mercados de Rusia y Estados Unidos.

\subsection{Posterior a análisis interno y externo $\rightarrow$ prospectiva visión a largo plazo}

Considerando que el direccionamiento de los procesos estratégicos en un sector depende, en gran medida, de la capacidad que se tenga para anticipar los cambios y construir de manera proac-

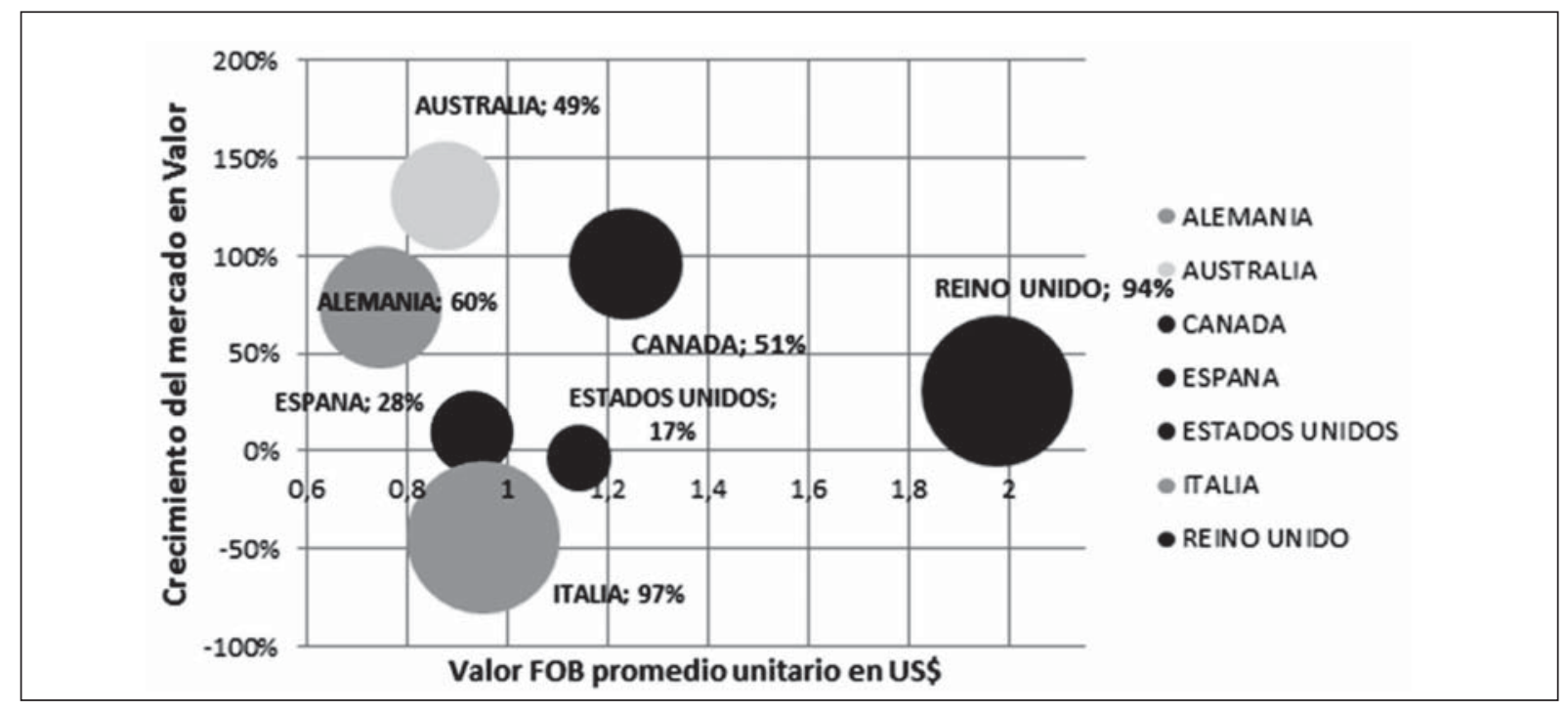

Figura 3. Identificación de los mercados reales y potenciales para panela pulverizada mercado por abarcar en valor. FOB (USD) unitario promedio frente a crecimiento del mercado en cantidad importada en kilogramos por país de destino. 2004-2007

Fuente: tomada de [3]. 


\section{investigación}

tiva el futuro; se formuló, a partir de los factores de menor previsibilidad, un conjunto de posibles escenarios futuros para la cadena productiva de la panela y su agroindustria a un horizonte de 10 años, es decir, el año 2019. Los tres escenarios formulados fueron: construyendo cadena en bloque con esfuerzos granulados, mieles tecnológicas y comerciales para moldear cadena y cadena en bloque, concentración tecnológica, mieles de comercialización y producto pulverizado; en cada uno de estos escenarios se articularon, de manera sistémica, aspectos de tipo tanto tecnológico como no tecnológico, siendo cada uno de ellos validado por actores del sector.

A partir de la caracterización de los tres escenarios posibles, se identificó cuál es el escenario apuesta o deseable para el sector en el año 2019, el reto que entonces se plantea, es generar, a partir de la agenda de investigación, estrategias que permitan pasar del escenario actual al escenario apuesta.

\section{CONCLUSIONES}

Agenda de investigación: desde el análisis de la cadena productiva, se realizó la identificación de oportunidades y limitaciones, las cuales fueron priorizadas, resultando de este modo los factores críticos de éxito, anteriormente mencionados, que a su vez dieron lugar a la identificación de demandas tecnológicas y no tecnológicas que constituyen una respuesta a cada uno de los factores críticos para el fortalecimiento de la competitividad.

La agenda surge, entonces, de un proceso de socialización y articulación con expertos de la cadena, quienes cuentan con una amplia trayectoria en el sector panelero, en el cual plantearon aquellos proyectos necesarios para suplir las once (11) demandas tecnológicas identificadas. Estas once demandas tecnológicas identificadas para la cadena de la panela y su agroindustria, están definidas para cuatro áreas estratégicas de trabajo investigativo y de desarrollo tecnológico:

1) Manejo sanitario y fitosanitario: en esta área se presentan las demandas o necesidades relacionadas con el manejo del cultivo frente a las plagas y enfermedades que afectan su sostenimiento y rendimiento. 2) Calidad e innovación de insumos y productos: en esta área se presentan las demandas relacionadas con el mejoramiento y desarrollo de productos e insumos para incrementar la productividad de la actividad de la cadena. 3) Manejo integral del cultivo: en esta área se integran las demandas referentes a las actividades del cultivo, desde la siembra hasta la cosecha. 4) Manejo poscosecha y transformación: en esta área se integran las demandas relacionadas con el manejo poscosecha de la caña y todas las etapas concernientes al proceso productivo de la fabricación de panela. En cada una de estas áreas estratégicas los expertos definieron en total 41 lineamientos en proyectos que permitirán fortalecer las once demandas tecnológicas identificadas en el estudio,

De manera homóloga a la forma que se identificaron las demandas tecnológicas, se abordó en el estudio la identificación de demandas no tecnológicas que dan soporte a la formulación de estrategias, para lineamientos organizacionales e institucionales que den soporte al cumplimiento de los objetivos de la agenda de investigación.

Para lograr la satisfactoria realización de esta agenda, se hace necesario continuar con un proceso de implementación que inicia con la definición del origen de los recursos para llevarla a cabo, y continúa con la identificación de responsables para la asignación de recursos, definición de prioridades y realización de seguimiento a su implementación, así como el planteamiento de los mecanismos efectivos de seguimiento de la agenda y su articulación con los diferentes lineamientos estratégicos (políticas, acuerdos, entre otras) de la cadena. 


\section{AGRADECIMIENTOS}

Al Ministerio de Agricultura y Desarrollo Rural MADR, a los especialistas del Proyecto Transición de la Agricultura del MADR los doctores Claudia Uribe y Gustavo Bernal, al Consejo Nacional Panelero, al Comité Técnico del Consejo, a la Coordinadora de la Cadena la Dra. Sofía Ortiz, al Secretario Técnico de la cadena el Ingeniero Fabio Roa Leguizamo, a los expertos que participaron en cada una de las etapas de construcción y validación de resultados del estudio, a los ingenieros Carlos Alberto Contreras y Diana Cristina Ramírez quienes participaron en este estudio.

\section{REFERENCIAS}

[1] FAO, Producción de Panela como estrategia de diversificación en la generación de ingresos en áreas rurales de América Latina, AGSF (Servicio de Gestión, Comercialización y FinanzasAgrícolas), Roma, 2004.

[2] FAO y G. Osorio, Manual Técnico Buenas Prácticas Agrícolas (BPA) y Buenas Prácticas de Manufactura (BPM) en la producción de caña y panela, Colombia, 2007.

[3] A. Rodríguez y Tibaitata, Evaluación de la adopción e impacto de la tecnología en la industria panelera y priorización de actividades futura de investigación y desarrollo, Corpoica CI, 2001

[4] O. Castellanos, Gestión Tecnológica: de un enfoque tradicional a la inteligencia, Universidad Nacional de Colombia, Bogotá, 2007.

[5] M. Porter, Ventaja competitiva, Compañía Editorial Continental S.A., México, 1987,

[6] N. Linares y Y. Salamanca, Determinación de la capacidad de interacción de las empresas en un ambiente competitivo. Caso: empresas biotecnológicas", [Trabajo de grado en administración de empresas], Bogotá, Universidad Nacional de Colombia, 2003.

[7] R. M. Bocchetto, (2001), Nuevas demandas y desafios del sistema agroalimentario y agroindustrial en el MERCOSUR ampliado: opciones para la integración tecnológica, [en línea]. Disponible: http:// webiica.iica.ac.cr/bibliotecas/repiica/ B0625E/B0625E.PDF

[8] P. Escorsa y R. M Maspons, De la vigilancia tecnológica a la inteligencia competitiva, Prentice Hall, 2001.

[9] COTEC, "Fundación para la innovación tecnológica. Vigilancia Tecnológica", en Documentos COTEC sobre oportunidades tecnológicas, España, COTEC, 1999.

[10] J. A. Martín, Prospectiva tecnológica: una introducción a su metodología y a su aplicación en distintos países, México, 2000.

[11] A. M. G. Castro y S. M. V, Lima, Análisis prospectivo de cadenas productivas agropecuarias, Empresa Brasileira de Pesquisas Agropecuarias, Perú, 2001.

[12] O. Castellanos, L. Torres y D. Florez, Agenda Prospectiva de investigación y desarrollo para la cadena productiva de la panela y su agroindustria en Colombia, Giro Editores. Bogotá, Ministerios de agricultura y desarrollo rural, 2010.

[13] O. Castellanos, Manual Metodológico Para la Definición de Agendas de Investigación $y$ desarrollo Tecnológico en Cadenas Productivas Agroindustriales, Universidad Nacional de Colombia. Bogotá, 2009. 